\title{
BIBLIOGRAPHIE PARTIELLE
}

\section{DES OEUVRES DE PHILOSOPHIE}

\section{EN LANGUE FRANÇAISE POUR L'ANNÉE 1990.}

Adam, Jean-Michel. Eléments de linguistique textuelle. Théorie et pratique de l'analyse textuelle. Liège: Pierre Margada, 1990.

Amiel, Anne. 50 grandes citations philosophiques expliquées. Les auteurs et les courants de pensée. Alleur: Marabout, 1990.

Ansart, Pierre. Les sociologies contemporaines. Ed. du Seuil, 1990.

Arnaud, Jean-Pierre. Wittgenstein, Freud, et la musique. La parole et le chant dans la communication. P.U.F., 1990.

Association des professeurs de philosophie de l'Académie de Poitiers. La Révolution francaise et la philosophie. Echanges et conflits. Poitiers, 1990.

Audouze, Jean, Paul Musset, Michel Paty (dir.). Les particules et lunivers. La rencontre de la physique des particules, de l'astrophysique et de la cosmologie. P.U.F., 1990.

Auffret, Dominique. Alexandre Kojève. La philosophie, l'Etat, la fin de l'histoire. Grasset, 1990.

Auroux, Sylvain. Barbarie et philosophie. P.U.F., 1990.

Badiou, Alain. Le Nombre et les nombres. Seuil, 1990.

Ballet, René. Lettres texanes. Messidor, 1990.

Barreau, Hervé. L'épistémologie. P.U.F., 1990.

Bastit, Michel. Naissance de la loi moderne. La pensée de la loi de saint Thomas à Suarez. P.U.F., 1990.

Bergson, Henri. Cours I : Leçons de psychologie et de métaphysique, Clermont-Ferrand 1887-1888. Édité par Henri Hude avec la collab. de Jean-Louis Dumas Avant-propos d'Henri Gouhier. P.U.F., 1990.

- Naissance d'une philosophie. Actes du colloque de ClermontFerrand (17 et 18 nov. 1989). P.U.F., 1990.

Berrêdo Carniero, Paulo E.'de. Auguste Comte. Correspondance générale et confessions. T. VIII : 1855-1857. Textes présentés par Angèle Kremer-Marietti. EHESS/Vrin, 1990. 
Berthelot, Jean-Michel. L'intelligence du social. Le Pluralisme explicatif en sociologie. P.U.F., 1990.

Bitbol-Hespériès, Annie. Le Principe de vie chez Descartes. Vrin, 1990.

Blanc-Ferret, François. Heureux les goélands. De la citoyenneté volontaire et de ses corollaires. Saint-Georges-sur-Eure, 1990.

Bloch, Olivier. Spinoza au XVIII' siècle. Méridiens-Klincksieck, 1990. Blondel, Maurice. Une dramatique de la modernité. Actes du colloque Maurice Blondel, Aix-en-Provence, mars 1989. Éd. Universitaires, 1990.

Boltanski, Luc. L'amour et la justice comme compétences. Trois essais de sociologie de l'action. Ed. Métailié, 1990.

Borch-Jacobsen, Mikkel. Lacan. Le maître absolu. Flammarion, 1990.

Borella, Jean. La Crise du symbolisme religieux. Lausanne: L'Age d'Homme, 1990.

Borrell, Joan. L'artiste-roi. Essais sur les représentations. Aubier, 1990.

Boss, Gilbert. John Stuart Mill. Induction et utilite. P.U.F., 1990.

Bossuet, Jacques-Bénigne. Introduction à la philosophie ou De la connaissance de Dieu et de soi-même. Fayard, 1990.

- Logique du Dauphin. Editions Universitaires, 1990.

Boudon, Raymond. L'art de se persuader des idées fausses, fragiles ou douteuses.... Fayard, 1990.

Bougnoux, Daniel, Jean-Louis Le Moigne et Serge Proulx. Arguments pour une méthode (autour d'Edgar Morin). Colloque de Cerisy. Éd. du Seuil, 1990.

Boulad-Ayoub, Josiane. Contre nous de la tyrannie.... Des relations idéologiques entre Lumières et Révolution. Hurtubise HMH ITTE, 1990. Bourgeois, Bernard. Philosophie et droits de l'homme. De Kant à Marx. P.U.F., 1990.

Boutroux, Emile. Leçons sur Aristote. Ed. critique et postface de Jérome de Gramont. Ed. Universitaires, 1990.

Brachet, Robert. L’âme religieuse du jeune Aristote. Paris-Fribourg: Ed. Saint-Paul, 1990.

Brenner, Anastasios. Duhem, sciences, réalité et apparence. La relation entre philosophie et histoire dans l'oeuvre de Pierre Duhem. Préf. de Maurice Boudot. Vrin, 1990.

Breton, Stanislas. Libres commentaires. Editions du Cerf, 1990.

Breuil, Paul du. Les Jains de l'Inde. Aubier, 1990.

Buci-Glucksmann, Christine. Tragique de l'ombre. Shakespeare et le 
maniérisme. Éd. Galilée, 1990.

Burggraeve, Roger. Emmanuel Levinas. Une bibliographie primaire et secondaire (1929-1985) avec complément 1985-1989. Leuven: Peeters, 1990.

Buydens, Mireille. Sahara. L'esthétique de Gilles Deleuze. Vrin, 1990. Canivez, Patrice. Eduquer le citoyen? Hatier, 1990.

Carlier, Pierre. Démosthène. Fayard, 1990.

Castillo, Monique. Kant et l'avenir de la culture. P.U.F., 1990.

Castoriadis, Cornelius. Le Monde morcelé. Les carrefours du labyrinthe III. Seuil, 1990.

Cauquelin, Anne. Aristote. Le langage. P.U.F., 1990.

Certeau, Michel de. L'Invention du quotidien. T.I: Arts de faire. Gallimard, 1990.

Champeau, Serge. Borges et la métaphysique. Vrin, 1990.

Chapouthier, Georges. Au bon vouloir de l'homme, l'animal. Denoël, 1990.

Charle, Christophe. Naissance des « intellectuels », 1880-1900. Ed. de Minuit, 1990.

Chédozeau, Bernard. La Bible et la liturgie en français. L'église tridentine et les traductions bibliques et liturgiques (1600-1789). Ed. du Cerf, 1990.

Chrétien, Jean-Louis. La Voix nue. Phénoménologie de la promesse. Minuit, 1990.

Coiffier, Eliane, et.al., Sociologie basique. Nathan, 1990.

Colliot-Thélène, Catherine. Max Weber et l'histoire. P.U.F., 1990.

Comte, Auguste. Correspondance générale et confessions, t. VIII: 18551857, éd. de Paulo E. de Berrêdo Carneiro, intr. d'Angèle Kremer-Marietti. Ed. de l'EHESS-Vrin, 1990.

Conche, Marcel. Orientation philosophique. P.U.F., 1990. - Le Fondement de la morale. Mégare, 1990.

Cortine, Jean-François. Heidegger et la phénoménologie. Vrin, 1990.

Cossutta, Frédéric. Éléments pour la lecture des textes philosophiques. Éd. Bordas, 1990.

Court, Raymond. Force et dérive des principes. Réflexions sur la raison moderne en procés. Méridiens Klincksieck, 1990.

Courtine, Jean-François. Suarez et le système de la métaphysique. P.U.F., 1990.

Covin, Michel. Une esthétique du sommeil. Beauchesne, 1990. 
Daelmans, Diane. La philosophie. Préliminaires: de la Renaissance à nos jours. Elvire Editions, 1990.

Dastur, Françoise. Heidegger et la question du temps. P.U.F., 1990.

Delaporte, François. Le savoir de la maladie. Essai sur le choléra de 1832 à Paris. P.U.F., 1990.

Deleuze, Gilles. Pourparlers, 1972-1990. Minuit, 1990.

Demont, Paul. La Cité grecque archaïque et classique et l'idéal de tranquillité. Les Belles Lettres, 1990.

Derrida, Jacques. Le probléme de la genèse dans la philosophie de Husserl. P.U.F., 1990.

Descamps, Christian. Les Enjeux philosophiques des années 1950. Centre Georges-Pompidou, 1990.

Descartes. Méditations métaphysiques. Méditationes de prima philosophia (Texte latin et traduction du duc de Luynes). Méditations de philosophie première. Présentation et traduction de Michelle Beyssade. L.G.F., 1990.

Devereux, Daniel, Pierre Pellegrin. Biologie, logique et métaphysique chez Aristote. Actes du Séminaire CNRS-NSF, Oléron, juinjuillet 1987. Ed. du CNHS, 1990.

Didi-Huberman, Georges. Devant l'image. Questions posées aux fins d'une histoire de l'art. Ed. de Minuit, 1990.

Dirn, Louis. La société française en tendances. P.U.F., 1990.

Droesbeke, Jean-Jacques, et Philippe Tassi. Histoire de la statistique. P.U.F., 1990.

Droit, Roger-Pol. Science et philosophie pour quoi faire? Le Monde, 1990.

Dubouchet, Paul. Méthodes des sciences sociales. Lyon, 1990.

Dufour-El Maleh, Marie-Cécile. Angelus novus. Essai sur l'oeuvre de Walter Benjamin. Bruxelles: Ed. Ousia, 1990.

Dumas,Jean-Louis. Histoire de la pensée. T. II : Renaissance et siècle des lumières. T. III : Temps modernes. Tallandier, 1990.

Dupuis, Pierre-André. Eduquer: une longue histoire. Recherches sur l'espace et le temps de l'éducation. Strasbourg: Presses Universitaires, 1990.

Durandeaux, Jacques. De l'éternité. Temps réfuté ou temps aimé? Desclée de Brouwer, 1990.

Duval, Raymond. Temps et vigilance. Vrin, 1990.

Duvernoy, Jean-François. L'épicurisme et sa tradition antique. Bordas, 
1990.

Echaudemaison, Claude-Danièle. Dictionnaire d'économie et de sciences sociales. Nathan, 1990.

Ellul, Jacques. La Technique ou l'Enjeu du siècle. Economica, 1990.

Espagnat, Bernard d'. Penser la science ou les enjeux du savoir. Dunod, 1990.

Etiemble, René. L'érotisme et l'amour. Arléa, 1990.

Everaert-Desmedt, Nicole. Le processus interprétatif. Introduction à la sémiotique de Ch. S. Peirce. Liège, 1990.

Faye, Jean-Pierre. La raison narrative. Langages totalitaires, Critique de l'économie narrative. Balland, 1990.

Felice, Domenico. Pour l'histoire de la fortune de Montesquieu en Italie (1789-1945). Bologne: Thema Editore, 1990.

Fénélon. Traité de l'existence de Dieu, éd. critique de Jean-Louis Dumas. Ed. Universitaires, 1990.

Ferry, Luc. Homo Aestheticus. L'invention du goût à l'âge démocratique. Grasset, 1990.

Foigny, Gabriel de. La Terre australe connue (1676). Éd. établie, présentée et annotée par Pierre Ronzeaud. S.T.F.M., 1990.

Fontenelle. Oeuvres complètes. t.l. Fayard, 1990.

Freund, Julien. Philosophie philosophique. La Découverte, 1990.

Gabaude, Jean-Marc. Un Demi-siecle de philosophie en langue française (1937-1990). Historique de l'Association des Sociétés de Philosophie de Langue française (ASPLF). Montreal: Ed. Montmorency, 1990.

Gaudin, Claude. Platon et l'alphabet. P.U.F., 1990.

Gérando, Joseph-Marie de. De la génération des connaissances humaines. Fayard, 1990.

Giovannangeli, Daniel. $\underline{\mathrm{La}}$ fiction de l'être. Lectures de la philosophie moderne. Bruxelles-Paris: De Boeck-Wesmael-Ed. Universitaires, 1990.

Goldschmidt, Vistor. Platonisme et pensée contemporaine. Vrin, 1990. Grand, Anne-Marie. Victor Ségalen. Le moi et l'expérience du vide. Méridiens/Klincksieck, 1990.

Granel, Gérard. Écrits logiques et politiques. Galilée, 1990.

Gravot, Robert. Conferences. Pascal. Peguy. Saint-John Perse. Teilhard de Chardin. Malraux. Imago Mundi. Brest-Paris: Ed. de la Cité, 1990. 
Grawitz, Madeleine. Michel Bakounine. Plon, 1990.

Gresle,François, Michel Panoff, Michel Perrin, Pierre Tripier.

Dictionnaire des sciences humaines. Sociologie, psychologie sociale, anthropologie. Nathan, 1990.

Grosz, Elizabeth. A feminist introduction. Routledge, 1990

Guérin, Michel. La Terreur et la pitié. T.I: la Terreur. Actes Sud, 1990.

Guy, Alain. Panorama de la philosophie ibéro-américaine. Du XVI siècle à nos jours. Patiño, 1990.

Haar, Michel. Heidegger et l'essence de l'homme. Grenoble, 1990.

Haldas, Georges. Carnets du désert. L'état de poésie. Carnets 1986. Age d'homme, 1990.

Halévy, Élie. L’ère des tyrannies. Études sur le socialisme et la guerre. Préf. de C. Bouglé. Postf. de Raymond Aron. Gallimard, 1990. Hassoun, Jacques, Christiane Riboni, Paul-Elie Lévy (dir.). Freud 18891989. Le voyage à Nancy. Nancy: Presses Universitaires de Nancy, 1990.

Hazard, Paul. La Pensée européenne au XVIII' siècle. De Montesquieu à Lessing. Fayard, 1990.

Hirschberger, Johannes. Abrégé d'histoire de la philosophie occidentale. Adaptation de Philibert Secretan. Fribourg (Suisse): Ed. Universitaires, 1990.

Hottois, Gilbert. Penser la logique. Une introduction technique, théorique et philosophique à la logique formelle. De BoeckWesmael/Ed. Universitaires, 1990.

- L'affect philosophe. Vrin, 1990.

Hude, Henri. Bergson. t.2. Editions Universitaires, 1990.

Huisman,Bruno et François Ribes. Les Philosophes et la nature. Bordas, 1990.

Illiez, Pierre. Reflets ou apparences. Causalité et incertitude dans I a pensée occidentale. Age d'homme, 1990.

Isambert-Jamati, Viviane. Les savoirs scolaires. Enjeux sociaux des contenus d'enseignement et de leurs réformes. Editions

Universitaires, 1990.

Ishaghpour, Youssef. Elias Canetti. Métamorphose et identité. La Différence, 1990.

Jaegle, Pierre et Pierre Roubaud. La Notion de la réalité. Messidor/Éd. Sociales, 1990.

Jambet, Christian. La Grande Résurrection d'Alamût. Les formes de la 
liberté dans le shîssime ismaïlien. Verdier, 1990.

Janicaud, Dominique. L'ombre de cette pensée. Heidegger et la question politique. Grenoble, 1990.

Jarczyk, Gwendoline. Éloges des libertés. Desclée de Brouwer, 1990.

Jeannière, Abel. Lire Platon. Aubier, 1990.

Kaplan, Francis. Marx antisémite? Imago/Berg international, 1990.

Kojève, Alexandre. L'Idée du déterminisme dans la physique classique

et dans la physique moderne. Présentation de Dominique Auffret. Librairie générale française, 1990.

- Le Concept, le temps et le discours. Introduction au système du savoir. Gallimard, 1990.

Korinman, Michel. Quand l'Allemagne pensait le monde. Grandeur et décadence d'une géopolitique. Fayard, 1990.

Labica, Georges. Robespierre. Une politique, la philosophie. P.U.F., 1990.

Lachelier, Jules. Cours de logique. École supérieure, 1866-1867. Ed. critique par Jean-Louis Dumas. Éd. Universitaires, 1990.

Lacoste, Jean-Yves. Note sur le temps. Essai sur les raisons de la mémoire et de l'espérance. P.U.F., 1990.

Laks, André et Ada Neschke. La Naissance du paradigme herméneutique. Schleiermacher, Humbolt, Boeckh, Droysen (vol. 10). Lille: P.U., 1990.

Lattre, Alain de. Bergson, une ontologie de la perplexité. P.U.F., 1990.

Lecourt, Dominique. Contre la peur. De la science à l'éthique, une aventure infinie. Hachette, 1990.

Ledrut, Raymond. Dits \& Inédits. P.U. du Mirail, 1990.

Lefebvre, Henri. Le matérialisme dialectique. P.U.F., 1990.

Lenoble, Jacques et André Berten. Dire la norme. Droit, politique et énonciation. Bruxelles-Paris: E. Story-Scientia-L.G.D.J., 1990.

Levinas, Emmanuel. Totalité et infini. Essai sur l'extériorité. Librairie Générale Française, 1990.

Lévy, Bernard-Henri. Question de principe trois. La suite dans les idées. Librairie Générale Française, 1990.

Lewitzky, Serge. Autonomie individuelle et appartenance au Tout. Deux principes, un concept. Académie européenne du Livre, 1990.

Libera, Alain de. Albert-le-Grand et la philosophie. Vrin, 1990.

Liger-Belair, Jean-Bernard. L'ombre nécessaire. Phénoménologie du corps. Ed. du Félin, 1990. 
Little, J.-P., et A. Ughetto. Simone Weil, la soif de l'absolu. Sud, 1990. Locke, John. Textes sur la loi de nature, la morale et la religion. Morale et loi naturelle. Présentation, traduction et notes de Jean-Fabien Spitz. Vrin, 1990.

Lurçat, François. Niels Bohr. Avant, aprés. Criterion, 1990.

Lurol, Gérard. Mounier, T. I : Genèse de la personne. Ed. Universitaires, 1990.

Macherey, Pierre. A quoi pense la littérature? Presses Universitaires de France, 1990.

Maïmon, Salomon. Essai sur la philosophie transcendantale. Présentation, traduction et notes par Jean-Baptiste Scherrer, avant-propos de Reinhard Lauth. Librairie philosophique J. Vrin, 1990.

Maine de Biran. Oeuvres, T. IX : Nouvelles considérations sur les rapports du physique et du moral de l'homme. Ecrits sur la physiologie, éd. par Bernard Baertschi. T. XI-1 Commentaires et marginalia : dix-septième siècle, éd. par Christaine Frémont. T. XI-3. Commentaires et marginalia : dix-neuvième siècle, éd. par Joël Ganault. Vrin, 1990.

Mannoni, Octave. Lettres personnelles. Fiction lacanienne d'une analyse. Denoël, 1990.

. Nous nous quittons. C'est là ma route. Carnets. Denoël, 1990.

Marion, Jean-Luc. Réduction et donation. Recherches sur Husserl, Heidegger et la phénoménologie. P.U.F., 1990.

Mathiot, Jean. Adam Smith. Philosophie et économie. De La sympathie à l'échange. P.U.F., 1990

Mattéi, Jean-François (dir.). La naissance de la raison en Grèce. P.U.F., 1990.

Mauzi, Robert (dir.). Précis de littérature française du XVIIIe siécle. P.U.F., 1990

McKenna, Antony. De Pascal à Voltaire. Le rôle des Pensées de Pascal dans l'histoire des idées entre 1670 et 1734 . Oxford: Voltaire Foundation, 1990.

Mendenhall, Vance. Une introduction à l'analyse du discours argumentatif. Des savoirs et savoir-faire fondamentaux. Ottawa: Les Presses de l'Université d'Ottawa, 1990.

Merleau-Ponty, Maurice. La structure du comportement; précédé de "Une philosophie de l'ambiguité » par Alphonse de Waelhens. P.U.F., 1990. 
Meschonnic, Henri. Le Langage Heidegger. P.U.F., 1990.

Meyer, Michel et Alain Lempereur (édit.). Figures et conflits rhétoriques. Bruxelles: Ed. de l'Université de Bruxelles, 1990.

Michon, Pierre. Maîtres et serviteurs. Verdier, 1990.

Minazzoli, Angés. La première ombre. Réflexion sur le miroir et la pensée. Ed. de Minuit, 1990.

Moles, Abraham A. et Elisabeth Rohmer. Les sciences de l'imprécis. Ed. du Seuil, 1990.

Monge, Jean. De la beauté ou La tentation de l'irrationnel. Toulouse: Ed. Universitaires du Sud, 1990.

Montet, Danielle. Les traits de l'être. Essai sur l'ontologie platonicienne. Grenoble: Ed. Jérôme Millon, 1990.

Montlibert, Christian de. Introduction au raisonnement sociologique, $2^{e}$ éd.aug. Strasbourg: P.U. de Strasbourg, 1990.

Morin, Edgar. Science avec conscience. Seuil, 1990. - Introduction à la pensée complexe. E.S.F., 1990.

Mourges, Jean. Le choix de l'homme. Une pensée maçonnique au quotidien. P.U.F., 1990.

Nicolet, Daniel. Lire Wittgenstein. Études pour une reconstruction fictive. Aubier, 1990.

Omesco, Ion. Othello, chef-d'oeuvre en sursis. Paris: P.U.F., 1990.

Onfray, Michel. Le ventre des philosophes. Critique de la raison diététique. Grasset, 1990.

- Cynismes. Portrait du philosophe en chien. Grasset, 1990.

Pariente, Jean-Claude (edit.). Bergson. Naissance d'une philosophie. Actes du colloque de Clermont-Ferrand, 17 et 18 novembre 1989. P.U.F., 1990.

Patocka, Jan. Liberté et sacrifice. Ecrits politiques. Grenoble: Jérôme Millon, 1990.

Perelman, Chaïm. Éthique et droit (T. III). Ed. de l'Université de Bruxelles, 1990.

Pharo, Patrick, Louis Quère (dir.). Les formes de l'action. Sémantique et sociologie. Ed. de l'Ecole des Hautes Etudes en

sciences sociales, 1990.

Philippe, Marie-Dominique. Lettre à un ami. Itinéraire philosophique. Ed. Universitaires, 1990.

Philonenko, Alexis. La Transcendantal et la pensée moderne. Études d'histoire de la philosophie. P.U.F., 1990. 
Pitassi, M.C. Le philosophe et l'écriture. John Locke exégète de S. Paul. Cahiers de la Revue de théologie et de philosophie, 14. GenèveLausanne-Neuchâtel: Revue de théologie et de philosophie,1990. Pomian, Krzysztof (édit.). La querelle du déterminisme. Philosophie de la science d'aujourd'hui. Gallimard, 1990.

Ponton, Lionel. Philosophic et droits de l'homme de Kant à Levinas. Vrin, 1990.

Prades, José A. Durkheim. P.U.F., 1990.

Proudhon, Pierre-Joseph. De la justice dans la Révolution et dans l'Eglise. Etudes de philosophie pratique. t. 3 et 4. Fayard, 1990.

Puech, Michel. Kant et la causalité. Etude sur la formation du système critique. Vrin, 1990.

Putnam, Hilary. Représentation et réalité. Trad. de Claudine EngelTiercelin. Gallimard, 1990.

Quillet, Jeannine. Autour de Nicole Oresme. Vrin, 1990.

Rancière, Jacques. Aux bords du politique. Editions Siris, 1990.

Revue philosophique de la France et de l'étranger, $n^{\circ} 2$ (1097), avril-juin 1990. "Derrida."

Richir, Marc. La crise du sens et la phénoménologie. Autour de la «Krisis » de Husserl. Suivi de Commentaire de « L'origine de la géométrie ». Grenoble, Jérôme Millon, 1990.

Ricoeur, Paul. Soi-même comme un autre. Éd. du Seuil, 1990.

Rivelaygue, Jacques. Leçons de métaphysique allemande. T.I: De Leibniz à Hegel. Grasset, 1990.

Robert, François. Les termes philosophiques. Alleur: Marabout, 1990. Rodis-Lewis, Geneviève. L'anthropologie cartésienne. P.U.F., 1990.

Roger, Philippe. Roland Barthes, roman. Grasset, 1990.

Romilly, Jacqueline de. La Construction de la vérité chez Thucydide. Julliard, 1990.

Rosello, Mireille. L'in-différence chez Michel Tournier. Jose Corti, 1990. Roustang, François. Influence. Ed. de Minuit, 1990.

Ruby, Christian. Histoire de la philosophie. La Découverte, 1990.

Ruelland, Jacques G. De l'épistémologie a la politique de Karl R. Popper. Presses Universitaires de France, 1990.

Ruyer, Bernard. Logique. Préf. de Jean Largeault. P.U.F., 1990. Saffrey, H.D. Recherches sur le néo-platonisme après Plotin. Vrin, 1990. Safranski, Rüdiger. Schopenhauer et les années folles de la philosophic. Une biographie. Trad. de l'allemand par Hans Hilden-brand, 
avec la collab. de Pierre Héber-Suffrin. P.U.F., 1990.

Saint-Martin, Louis-Claude de. Controverse avec Garat précédée d'autres écrits philosophiques. Fayard, 1990.

Salem, Jean. La mort n'est rien pour nous. Lucrece et l'ethique. Vrin, 1990.

. Tel un Dieu parmi les hommes. L'éthique d'Épicure. Vrin, 1990.

Sans, Édouard. Schopenhauer. P.U.F., 1990.

Sartre, Jean-Paul. Situations philosophiques. Gallimard, 1990.

Schlanger, Jacques. La situation cognitive. Méridiens-Klincksieck, 1990.

Seidengart, Jean. Ernst Cassirer. De Marbourg à New York. L'itinéraire philosophique. Ed. du Cerf, 1990.

Serres, Michel. Le Contrat naturel. Éd. François Bourin, 1990.

Seve, Lucien. Le communisme. Quel second souffle? Messidor/Ed. Sociales, 1990.

Sichère, Bernard. Eloge du sujet. Du retard de la pensée sur les corps. Grasset, 1990.

Société toulousaine de Philosophie. Présence de Georges Bastide. Actes du colloque Georges Bastide (1901-1969). "Appartenance et présence d'un philosophe français." Toulouse: Editions universitaires du Sud, 1990.

Sorel, Georges. Réflexions sur la violence. Éd. établie par Michel Prat. Préf. de Jacques Julliard. Éd. du Seuil, 1990.

Souche-Dagues, Denise. Hégelianisme et dualisme. Réflexions sur le phénomène. Vrin, 1990.

Terray, Emmanuel. La Politique dans la caverne. Éd. du Seuil, 1990.

Theau, Jean. Trois essais sur la pensée. Ottawa: Les Presses de l'Université d'Ottawa, 1990.

Thom, René. Apologie du logos. Hachette, 1990.

Thomas, Jean-Paul. Misère de la bióthique. Pour une morale contre les apprentis sorciers. Albin Michel, 1990.

Trigeaud, Jean-Marc. Persona ou la justice au double visage. Gênes: Studio Editoriale di Cultura, 1990.

- Humanisme de la liberté et philosophie de la justice. T. 2. Bordeaux, 1990.

Trotignon, Pierre. Les chemins du philosophe. Fayard, 1990.

Vacher, Laurent-Michel. L'empire du moderne. Actualité de la philosophie américaine. Montréal: Les Herbes rouges, 1990. 
Valdinoci, Serge. Introduction dans l'Europanalyse. Krisis 2 : Transformer la phénoménologie de Husserl pour fonder la philosophie. Augier, 1990.

Vassilie-Lemeny, Sorin-Titus. Pour une philosophie du sens et de la valeur. Bordeaux: Ed. Brière, 1990.

Védrine, Hélène. Les grandes conceptions de l'imaginaire de Platon à Sartre et Lacan. Librairie générale française, 1990.

Vernant, Jean-Pierre. Figures, idoles, masques. Julliard, 1990.

Viry, Robert. Introduction à l'étude de la psychiatrie analytique. Nancy: Presses Universitaires de Nancy, 1990.

Voelke, André-Jean. Le scepticisme antique. Perspectives historiques et systématiques. Lausanne: Cahiers de la Revue de théologie et de philosophie, 1990.

Walch, Jean. Historiographie structurale. Masson, 1990.

Wallon, Henri. Psychologie et dialectique. La spirale et le miroir. Ecrits de 1926 à 1961, choisis et présentés par Emile Jalley et Liliane Maury. Messidor-Editions sociales, 1990.

Wijayaratna, Môhan. Le Bouddha et ses disciples. Texte intégral de 27 textes du « Canon bouddhique » Préf. d'André Padoux. Cerf, 1990.

Wunenburger, Jean-Jacques. La raison contradictoire. Sciences et philosophie modernes: la pensée du complexe. Albin Michel, 1990.

Zarader, Marléne. La dette impensée: Heidegger et l'héritage hébraique. Ed. du Seuil, 1990.

Zarka, Yves Charles. Thomas Hobbes. Philosophie première, théorie de la science et politique. P.U.F., 1990. 